\title{
The Series of Semigroup Theory Via Functional Calculus
}

\author{
Simon Joseph1*, Isra Mukhtar², Manal Juma ${ }^{3}$
}

${ }^{1}$ University of Juba, Faculty of Education, Department of Mathematics, South Sudan, Juba.

${ }^{2}$ Shaqra University, College of Science and Humanities (Girl Branch), Department of Mathematics, Al-Riyadh.

${ }^{3}$ Prince Sattam bin Abdulaziz University, College of Science and Humanity Studies (Girls Sections),

Department of Mathematics, Kingdom of Saudi Arabia, Alkharj.

University of Nyla, Faculty of Education, Department of Mathematics, Sudan.

Corresponding Author: Simon Joseph, University of Juba, Faculty of Education, Department of Mathematics, South Sudan, Juba. Email: s.j.u.khafiir@gmail.com

Mobile: +211954549999, +249912585607, Fax: +249925122242, +211912987666.

Abstract: Present panorama of the sequence of operators classes with their associated functional calculi, relevant in semi group theory: the sequence of operators of half plane, strip, sector and parabola-type. It is shown that the basic results in the theory of $C_{0}$-semigroup (the Hille-Yosida and the Trotter-kato theorem) follow easily from general functional calculus principles by Markus Haase [9]. The introduction of parabola-type sequence of operators allows to treat cosine the sequence of operator's functions by functional calculus methods.

Keywords and Phrase: $C_{0}$-semigroup, Functional calculus, Hille - Yosida, Trotter - Kato.

\section{INTRODUCTION}

The theory of strongly continuous study with the fundamental result of Hille and Yosida dating back to 1948. Several monographs and textbooks cover the by canonical material, each of them having its own peculiar point of view. One may focus entirely on the semigroup, and consider the generator as a derived concept (as in [3]) or one may start with the generator and view the semigroup as the pre-Laplace transform of the resolvent (as in [1]).

On the other side, during the last two decades the theory of functional calculus has proved to be an indispensable tool to deal with abstract evolution equations, above all in the discussion of maximal regularity. Despite their success, these methods have been mainly restricted to sectorial operators and hence to holomorphic semigroups. Eventually, $C_{0}$-groups were covered by a functional calculus for strip type operators [4]; but this was done because of the prominent role the groups of imaginary powers of a sectorial operator play in the parabolic world by Markus Haase [9]. Anyway, an approach to general semigroups via holomorphic functional calculus is missing up to now, as well as one for cosine operator functions (In fact that it was not clear for some time whether cosine functions could be treated by functional calculus method at all) .

Want to close this gap. We complement the existing holomorphic functional calculi (for sectorial and strip-type operators) by two more, one on halfplanes and one on parabolas. (The first covers general semigroups, the second cosine functions.) The strength of this approach lies in the fact that do not have to require the operator to generate a semigroup or a cosine function, but can work just with a certain natural growth condition on the norm of the resolvent. As a conseqence can give a short and straightfoward proof of the Hille-Yosida theorem, based only on one of the cornerstones of general functional calculus theory, the so-called Convergence Lemma A similar remark applies to the Trotter-Kato theorem.

So it turns out that the theory functional calculus indeed provides a valid starting point for general semigroup theory, not only in the holomorphic case.

\section{A Functional Calculus on the Half-Plane}

Let $A_{j}$ be the sequence of generators of $C_{0}$-semigroup $\left(T^{j}(1+\varepsilon)\right)_{(1+\varepsilon) \in \mathbb{R}}$ on a Banach space X. Would like to interpret $T^{j}(1+\varepsilon)=\left(e^{(1+\varepsilon) z}\right)\left(A_{j}\right),(\varepsilon>-1)$, 
were $e^{(1+\varepsilon) z}$ denotes the complex function $\mathrm{z} \rightarrow e^{(1+\varepsilon) z}$, and this should only be a special case of a general set-up in which " $f_{j}\left(A_{j}\right)$ " are defined in a reasonable way for as much functions $f_{j}$ as possible. The approach is based on the Cauchy integral formula; as we shall see, this to be viable requires only knowledge of the resolvent outside the sequence of spectrums of $A_{j}$. (In particular, the generator property is not necessary to construct the functional calculus in the first place.) As the procedure should work for all semigroup generators, lead to a functional calculus on (left) half-planes.

Let us fix some notation. For $(1+\varepsilon) \in[-\infty, \infty]$ define

$L_{(1+\varepsilon)}:=\{\mathrm{z} \in \mathbb{C} \mid \operatorname{Re} \mathrm{z}<(1+\varepsilon)\}$,

$R_{(1+\varepsilon)}:=\{\mathrm{z} \in \mathbb{C} \mid \operatorname{Re} \mathrm{z}>(1+\varepsilon)\}$

The open left and right half-planes defined by the abscissa $\operatorname{Re} z=(1+\varepsilon)$, where in the extremal cases one half-plane is understood to be empty, and the other is the whole complex plane.

Say that the sequence of operators $A_{j}$ on a Banach space $\mathrm{X}$ is of half-plane type

$(1+\varepsilon) \in \mathbb{R} \cup\{-\infty\}$, if $R_{(1+\varepsilon)} \subset \mathrm{e}\left(A_{j}\right)$ and

$M_{(\beta+\varepsilon)}:=M_{(\beta+\varepsilon)}\left(A_{j}\right):=\sup \left\{\mathrm{R}\left(\mathrm{z}, A_{j}\right) \mid \operatorname{Re} \mathrm{z} \geq \beta+\varepsilon\right\}<\infty$

for every $\beta+\varepsilon>(1+\varepsilon)$. We call

$(1+\varepsilon)_{0}\left(A_{j}\right):=\min \left\{(1+\varepsilon) \mid A_{j}\right.$ is of half-plane type $\left.(1+\varepsilon)\right\} \in[-\infty, \infty]$

The abscissa of uniform boundedness (in short: the aub) of the sequence of operators $A_{j}$.

Since all notions are invariant under translations parallel to the real axis, assume in the following that $(1+\varepsilon)_{0}\left(A_{j}\right) \leq 0$. Fix $0<\varepsilon<\infty$ and define

$\mathcal{E}\left(L_{(1+\varepsilon)}\right):=\left\{f_{j}: L_{(1+\varepsilon)} \rightarrow \mathbb{C} \mid f_{j}\right.$ is holomorphic and

$\exists \mathrm{M}, \varepsilon>-1:\left|f_{j}(\mathrm{z})\right| \leq \mathrm{M}|z|^{-(2+\varepsilon)}$ as $\left.\mathrm{z} \rightarrow \infty\right\}$

By standard applications of Cauchy's integral theorem for $f_{j} \in \mathcal{E}\left(L_{(1+\varepsilon)}\right)$ the formula

$$
\sum_{j} f_{j}(a)=\frac{1}{2 \pi i} \int_{\operatorname{Re} z=(1+\varepsilon)} \sum_{j} f_{j}(z) \frac{d z}{z-a}(\operatorname{Re} a \leq 0)
$$

holds. Here $(1+\varepsilon) \in(0,(1+\varepsilon))$ is arbitrary, and the direction of integration is bottom up,i.e., from $((1+\varepsilon)-\mathrm{i} \infty)$ to $((1+\varepsilon)+\mathrm{i} \infty)$. Since the resolvent $\mathrm{R}\left(\cdot, A_{j}\right)$ is bounded on the vertical lines

$(\operatorname{Re} \mathrm{z}=(1+\varepsilon)), \varepsilon>-1$, the integral

$$
\sum_{j} \Phi\left(f_{j}\right)=\sum_{j} f_{j}\left(A_{j}\right)=\frac{1}{2 \pi i} \int_{R e z=(1+\varepsilon)} \sum_{j} f_{j}(z) R\left(z, A_{j}\right)
$$

converges absolutely.

\section{Proposition 2.1}

The so defined mapping $\Phi: \mathcal{E}\left(L_{(1+\varepsilon)}\right) \rightarrow \mathcal{L}(\mathrm{X})$ satisfies the following properties:

a) $\Phi$ is a homomorphism of algebras.

b) If $T^{j} \in \mathcal{L}(\mathrm{X})$ commutes with $A_{j}$, i.e., $T^{j} A_{j} \subset A_{j} T^{j}$, it commutes with every $\Phi\left(f_{j}\right), f_{j} \in \mathcal{E}$.

c) $\Phi\left(f_{j}(\mathrm{z})((1+\varepsilon)-\mathrm{z})^{-1}\right)=\Phi\left(f_{j}\right) \mathrm{R}\left((1+\varepsilon), A_{j}\right)$ for all $\operatorname{Re}(1+\varepsilon)>(1+\varepsilon)$.

d) $\Phi\left(((1+\varepsilon)-\mathrm{z})^{-1}\left(\mu^{j}-\mathrm{z}\right)^{-1}\right)=\mathrm{R}\left((1+\varepsilon), A_{j}\right) \mathrm{R}\left(\mu^{j}, A_{j}\right)$ for all $\operatorname{Re}(1+\varepsilon), \operatorname{Re} \mu^{j}>(1+\varepsilon)$. 


\section{Proof}

a) This follows from a combination of Fubini's theorem, the resolvent identity and Cauchy's theorem. The computation is the same as in the classical Dunford-Rieszsetting, see [2, VII.4.7].

b) is obvious.

c) follows from the resolvent identity, since by Cauchy's theorem the integral

$\int_{\operatorname{Rez}-(1+\varepsilon)} f_{j}(\mathrm{z}) /((1+\varepsilon)-\mathrm{z}) \mathrm{dz}$ is equal to zero.

d) Here a standard path deformation argument is used. In the integral

$\int_{R e z-(1+\varepsilon)}((1+\varepsilon)-\mathrm{z})^{-1}\left(\mu^{j}-\mathrm{z}\right)^{-1} \mathrm{R}\left(\mathrm{z}, A_{j}\right) \mathrm{dz}$ shift the path to the right, i.e., let $(1+\varepsilon)$ grow. When passing the bscissas $(1+\varepsilon)=\operatorname{Re}(1+\varepsilon)$ and $(1+\varepsilon)=\operatorname{Re} \mu^{j}$ the residue theorem yields some additive contributions which sum up to $\mathrm{R}\left((1+\varepsilon), A_{j}\right) \mathrm{R}\left(\mu^{j}, A_{j}\right)$ by the resolvent identity; if $(1+\varepsilon)>\operatorname{Re}(1+\varepsilon)$, $\operatorname{Re} \mu^{j}$, the integral does not change any more as $(1+\varepsilon) \gamma_{\infty}$ and hence it is equalto zero.

Denote by $\mathcal{M}\left(L_{(1+\varepsilon)}\right)$ the field of meromorphic functions on the left half-plane Rez $<(1+\varepsilon)$. Then the triple $\left(\mathcal{E}\left(L_{(1+\varepsilon)}\right), \mathcal{M}\left(L_{(1+\varepsilon)}\right), \Phi\right)$ is a meromorphic functional calculusin the sense of [7, Section 1.3]. A meromorphic functions $f_{j}$ is called regularisable ifthere is a function e $\in \mathcal{E}$ such that e $f_{j} \in \mathcal{E}$ and $\mathrm{e}\left(A_{j}\right)$ is injective. In this case one defines $f_{j}\left(A_{j}\right):=\mathrm{e}\left(A_{j}\right)^{-1}\left(\mathrm{e} f_{j}\right)\left(A_{j}\right)$, which is a closed sequence operators. This definition does not depend on the chosen regulariser e $\in \mathcal{E}\left(\mathrm{c} f_{j}\right.$. [7, Section .1.2.1]).

The basic rules governing this functional calculus are the same as for any meromorphic functional calculus, see [7]. The two most important of these are the laws for sums:

$f_{j}\left(A_{j}\right)+\mathrm{g}^{j}\left(A_{j}\right) \subset\left(f_{j}+\mathrm{g}^{j}\right)\left(A_{j}\right)$

and products

$f_{j}\left(A_{j}\right) \mathrm{g}^{j}\left(A_{j}\right) \subset\left(f_{j} \mathrm{~g}^{j}\right)\left(A_{j}\right), \mathcal{D}\left(\left(f_{j} \mathrm{~g}^{j}\right)\left(A_{j}\right)\right) \cap \mathcal{D}\left(\mathrm{g}^{j}\left(A_{j}\right)\right)=\mathcal{D}\left(f_{j}\left(A_{j}\right) \mathrm{g}^{j}\left(A_{j}\right)\right)$.

In particular, one has $f_{j}\left(A_{j}\right)+\mathrm{g}^{j}\left(A_{j}\right)=\left(f_{j}+\mathrm{g}\right)\left(A_{j}\right)$ and $f_{j}\left(A_{j}\right) \mathrm{g}^{j}\left(A_{j}\right)=\left(f_{j} \mathrm{~g}^{j}\right)\left(A_{j}\right)$ whenever $\left(A_{j}\right) \in \mathcal{L}(\mathrm{X})$.

Note that every bounded analytic functions $f_{j} \in H^{\infty}\left(L_{(1+\varepsilon)}\right)$ is regular is able, namely by the function $\mathrm{e}(\mathrm{z}):=(1-\mathrm{z})^{-2}$. This is because $f_{j}(\mathrm{z})(1-\mathrm{z})^{-2}$ decreases quadratially as $\mathrm{z} \rightarrow \infty$ and $\mathrm{e}\left(A_{j}\right)=\mathrm{R}\left(1, A_{j}\right)^{2}$ is clearly injective. In particular, for each $\varepsilon>-1$ the sequence of operators $e^{(1+\varepsilon) A_{j}}:=\left(e^{(1+\varepsilon) z}\right)\left(A_{j}\right)$ is defined as a closed sequence of operators and $\mathcal{D}\left(A_{j}^{2}\right) \subset \mathcal{D}\left(e^{(1+\varepsilon)(1+\varepsilon)}\right), \varepsilon>-1$.

\section{Lemma 2.2}

Let $A_{j}$ be the sequence of operators of half-plane type, with $(1+\varepsilon)_{0}\left(A_{j}\right) \leq 0$. Then for each $\mathrm{x} \in \mathcal{D}\left(A_{j}^{2}\right)$ the function $\left((1+\varepsilon) \rightarrow e^{-(1+\varepsilon)(1+\varepsilon)} e^{(1+\varepsilon) A_{j}} \mathrm{x}\right):[0, \infty) \rightarrow \mathrm{X}$ is continuous and bounded, and its Laplace transform is

$$
\int_{0}^{\infty} e^{-(1+\varepsilon)(1+\varepsilon)} \sum_{j} e^{(1+\varepsilon) A_{j}} \mathrm{xd}(1+\varepsilon)=\mathrm{R}\left((1+\varepsilon), A_{j}\right) \mathrm{x}(\operatorname{Re}(1+\varepsilon)>(1+\varepsilon))
$$

\section{Proof}

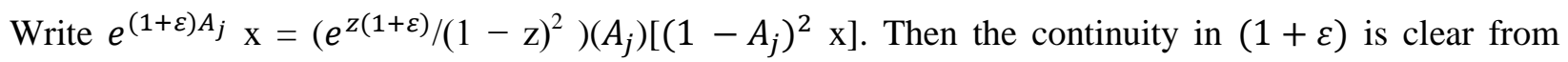
Lebesgue's theorem. The rest follows by Fubini's theorem.

Lemma 2.3

Let $A_{j}$ be a densely defined sequence of operators of half-plane type. Then $\mathcal{D}\left(A_{j}^{2}\right)$ are dense. 


\section{Proof}

For $\mathrm{x} \in \mathcal{D}\left(A_{j}\right)$ and $\mathrm{n} \in \mathrm{N}$ we write $\mathrm{nR}\left(\mathrm{n}, A_{j}\right) \mathrm{x}=\mathrm{x}+\mathrm{R}\left(\mathrm{n}, A_{j}\right)\left(A_{j}\right) \mathrm{x}$. The right hand side is bounded in $\mathrm{n}$, hence $\mathrm{R}\left(\mathrm{n}, A_{j}\right) \mathrm{x} \rightarrow 0$ as $\mathrm{n} \rightarrow \infty$. But $\mathcal{D}\left(A_{j}\right)$ is dense and the sequence of operators $\left(\mathrm{R}\left(\mathrm{n}, A_{j}\right)\right)_{\mathrm{n} \geq 1}$ are uniformly bounded. Hence $\mathrm{R}\left(\mathrm{n}, A_{j}\right) \mathrm{x} \rightarrow 0$ for all $\mathrm{x} \in \mathrm{X}$. But this implies that $\mathrm{nR}\left(\mathrm{n}, A_{j}\right) \mathrm{x}=\mathrm{x}+\mathrm{R}\left(\mathrm{n}, A_{j}\right) \mathrm{Ax}$ $\rightarrow \mathrm{x}$ whenever $\mathrm{x} \in \mathcal{D}\left(A_{j}\right)$;so $\mathcal{D}\left(A_{j}\right) \subset \overline{\mathcal{D}\left(A_{\mathrm{j}}^{2}\right)}$ which implies that $\mathcal{D}\left(A_{j}^{2}\right)$ is dense in $\mathrm{X}$.

\section{Proposition 2.4}

Let $A_{j}$ be sequence of operators of half-plane type, with $(1+\varepsilon)_{0}\left(A_{j}\right) \leq 0$. Then $A_{j}$ is the sequence of generators of a $\mathrm{C}_{0}$-semigroup $T^{j}$ if and only if $A_{j}$ is densely defined and $e^{\left((1+\varepsilon)_{1}+\cdots+(1+\varepsilon)_{n}\right) A_{j}}$ is a bounded sequence of operators for all $(1+\varepsilon)_{1}+\cdots+(1+\varepsilon)_{n} \in[0,1]$ satisfying $\sup (1+\varepsilon)_{1}+\cdots+(1+\varepsilon)_{n} \in$ $[0,1] e^{\left((1+\varepsilon)_{1}+\cdots+(1+\varepsilon)_{n}\right) A_{j}<\infty}$. In this case,

$T^{j}\left((1+\varepsilon)_{1}+\cdots+(1+\varepsilon)_{n}\right)=e^{\left((1+\varepsilon)_{1}+\cdots+(1+\varepsilon)_{n}\right) A_{j}}=\prod_{i=1}^{n} e^{(1+\varepsilon)_{i} A_{j}}$ for all $1 \leq i \leq n$

Proof

Let $A_{j}$ sequence of generates $\mathrm{C}_{0}$-semigroup $\left(T^{j}\left((1+\varepsilon)_{1}+\cdots+(1+\varepsilon)_{n}\right)\right)_{(1+\varepsilon)_{1}+\cdots+(1+\varepsilon)_{n} \geq 0}$. Then $A_{j}$ is densely defined. Hence $\mathcal{D}\left(A_{j}^{2}\right)$ is dense, by Lemma 2.3 Lemma 2.2 yields that $\mathrm{R}\left(\cdot, A_{j}\right) \mathrm{x}$ is the Laplace transform of $e^{A_{j}} \mathrm{x}$ for $\mathrm{x} \in \mathcal{D}\left(A_{j}^{2}\right)$. By the uniqueness of Laplace transforms, $T^{j}\left((1+\varepsilon)_{1}+\cdots+(1+\right.$ $\varepsilon) n) \mathrm{x}=e(1+\varepsilon) A \dot{\mathrm{x}},(1+\varepsilon) 1+\cdots+(1+\varepsilon)) n \geq 0$. Since $\mathcal{D}(A j 2)$ is dense and $e((1+\varepsilon) 1+\cdots+(1+\varepsilon) n) A j$ are closed sequence of operators, $e^{\left((1+\varepsilon)_{1}+\cdots+(1+\varepsilon)_{n}\right) A_{j}}=T^{j}\left((1+\varepsilon)_{1}+\cdots+(1+\varepsilon)_{n}\right)$ are abounded sequence of operators.

Conversely, suppose that $A_{j}$ is densely defined and $T^{j}(1+\varepsilon)_{1}+\cdots+(1+\varepsilon)_{n}:=e^{\left((1+\varepsilon)_{1}+\cdots+(1+\varepsilon)_{n}\right) A_{j}}$ are bounded sequence of operators for all $(1+\varepsilon)_{1}+\cdots+(1+\varepsilon)_{n} \geq 0$. Then $T^{j}$ are semigroup (by general functional calculus) and $\mathcal{D}\left(A_{j}^{2}\right)$ is dense, by Lemma 2.2. From the uniform boundedness $\sup _{(1+\varepsilon)_{1}+\cdots+(1+\varepsilon)_{n \in[0,1])}} T^{j}\left((1+\varepsilon)_{1}+\cdots+(1+\varepsilon)_{n}\right)<\infty$ one concludes easily that $\left(T^{j}((1+\varepsilon)+\right.$ $\left.\left.\cdots+(1+\varepsilon)_{n}\right)\right)$ is uniformly bounded on compact intervals. Lemma 2.2 and the density of $\mathcal{D}\left(A_{j}^{2}\right)$ imply that $\left(T^{j}\left((1+\varepsilon)_{1}+\cdots+(1+\varepsilon)_{n}\right)\right)$ is strongly continuous. Its Laplace transform coincides with the resolvent of $A_{j}$ on $\mathcal{D}\left(A_{j}^{2}\right)$ (Lemma 2.2, hence on $\mathrm{X}$ by density. So $A_{j}$ are the sequence of generators of $T^{j}$

\section{The Hille-Yosida TheOREM}

The Hille-Yosida theorem is one of the most fundamental results in the "elemen-tary" theory of $C_{0}$ semigroups. Show that it is a straightforward consequence of the following general fact of functional calculus theory (see, e.g.,[9]) .

\section{Proposition 3.1}

(Convergence Lemma) Let $A_{j}$ be a densely defined half-plane type of sequence of operators on the Banach space $\mathrm{X}$, with $(1+\varepsilon)_{0}\left(A_{j}\right) \leq 0$. Let $0<\varepsilon<\infty$ and let $\left(\left(f_{j}\right)_{i}\right)_{i}$ be net in $H^{\infty}\left(L_{(1+\varepsilon)}\right)$ satisfying the following conditions:

(i) $\sup _{i} \sum_{j}\left\|\left(f_{j}\right)_{i}\right\|_{\infty}<\infty$;

(ii) $\left(f_{j}\right)_{i}\left(A_{j}\right) \in \mathcal{L}(\mathrm{X})$ for all i, and $\sup _{i} \sum_{j}\left\|\left(f_{j}\right)_{i}\left(A_{j}\right)\right\|<\infty$;

(iii) $f_{j}(\mathrm{z}):=\lim _{i}\left(f_{j}\right)_{i}(\mathrm{z})$ exists for every $\mathrm{z} \in L_{(1+\varepsilon)}$.

Then $f_{j} \in H^{\infty}\left(L_{(1+\varepsilon)}\right), f_{j}\left(A_{j}\right) \in \mathcal{L}(\mathrm{X}),\left(f_{j}\right)_{i}\left(A_{j}\right) \rightarrow f_{j}\left(A_{j}\right)$ strongly, and

$\sum_{j}\left\|f_{j}\left(A_{j}\right)\right\| \leq \limsup i\left\|\sum_{j}\left(f_{j}\right)_{i}\left(A_{j}\right)\right\|$ 


\section{Proof.}

The proof is analogous to the proof of [7, Proposition 5.1.4]. By Vitali'stheorem, $f_{j}$ are holomorphic and the convergence of the $\left(f_{j}\right)_{i}$ to $f_{j}$ is uniform on compacts. Moreover, condition (i) clearly implies that $f_{j}$ is bounded. By Lebesgue's theorem and the definition of the elementary functional calculus

$\left(\left(f_{j}\right)_{i}(\mathrm{z})(1-\mathrm{z})^{-2}\right)\left(A_{j}\right) \rightarrow\left(f_{j}(\mathrm{z})(1-\mathrm{z})^{-2}\right)\left(A_{j}\right)$ in norm. Hence for

$\mathrm{x} \in \mathcal{D}\left(A_{j}^{2}\right),\left(f_{j}\right)_{i}\left(A_{j}\right) \mathrm{x}=\left(\left(f_{j}\right)_{i}(\mathrm{z})(1-\mathrm{z})^{-2}\right)\left(A_{j}\right)\left(1-A_{j}\right)^{2} \mathrm{x} \rightarrow\left(f_{j}(\mathrm{z})(1-\mathrm{z})^{-2}\right)\left(A_{j}\right)\left(1-A_{j}\right)^{2} \mathrm{x}=f_{j}\left(A_{j}\right) \mathrm{x}$. Clearly $\sum_{j}\left\|f_{j}\left(A_{j}\right) \mathrm{x}\right\| \leq \lim \sup _{i}\left\|\sum_{j}\left(f_{j}\right)_{i}\left(A_{j}\right)\right\|$. Since $f_{j}\left(A_{j}\right)$ is a closed sequence of operators with dense domain $\mathcal{D}\left(f_{j}\left(A_{j}\right)\right) \supset \mathcal{D}\left(A_{j}^{2}\right), f_{j}\left(A_{j}\right)$ is bounded with $\sum_{j}\left\|f_{j}\left(A_{j}\right)\right\| \leq$

$\limsup _{i}\left\|\sum_{j}\left(f_{j}\right)_{i}\left(A_{j}\right)\right\|$. Again by the density of $\mathcal{D}\left(A_{j}^{2}\right),\left(f_{j}\right)_{i}\left(A_{j}\right) \rightarrow f_{j}\left(A_{j}\right)$ strongly.

Theorem 3.2

(Hille-Yosida) Let $A_{j}$ be a densely defined sequence of operators on the Banach space X such that

$(0, \infty) \subset \varrho\left(A_{j}\right)$ and $\mathrm{M}:=\sup _{n \in N, \varepsilon>-1}\left\|\left[(1+\varepsilon) \mathrm{R}\left((1+\varepsilon), A_{j}\right)\right]^{n}\right\|<\infty$. Then $A_{j}$ is of half-plane type with $(1+\varepsilon)_{0}\left(A_{j}\right) \leq 0$ and $\left\|e^{(1+\varepsilon) A_{j}}\right\| \leq \mathrm{M}$ for all $\varepsilon>-1$.

Proof.

First show that $A_{j}$ is of half-plane type. Fix $\mu^{j}$ such that $\operatorname{Re} \mu^{j}>0$. For $\varepsilon>-1$ large, more precisely for $(1+\varepsilon)>\left|\mu^{j}\right|^{2} /\left(2 \operatorname{Re} \mu^{j}\right)$, one has $\left|(1+\varepsilon)-\mu^{j}\right|<(1+\varepsilon)$. By the Laurent series expansion of the resolvent,

$$
\sum_{j} R\left(\mu^{j}, A_{j}\right)=\sum_{j} \sum_{n=0}^{\infty}\left(\mu^{j}-(1+\varepsilon)\right)^{n} R\left((1+\varepsilon), A_{j}\right)^{n+1}
$$

and hence $\sum_{j}\left\|R\left(\mu^{j}, A_{j}\right)\right\| \leq M\left|\sum_{j} \mu^{j}-(1+\varepsilon)\right|^{n} /(1+\varepsilon)^{n+1}=M /\left((1+\varepsilon)-\mid \sum_{j} \mu^{j}-(1+\varepsilon)\right) \mid$. Let $(1+\varepsilon) \rightarrow \infty$ to conclude $\left\|\mathrm{R}\left(\mu^{j}, A_{j}\right)\right\| \leq M / \operatorname{Re} \mu^{j}$. It follows that $A_{j}$ is of half-plane type with $(1+\varepsilon)_{0}$ $\left(A_{j}\right) \leq 0$.

Define $r_{n,(1+\varepsilon)}(z):=(1-((1+\varepsilon) \mathrm{z}) / \mathrm{n})^{-n}$. For fixed $0<\varepsilon<\infty$ and large $\mathrm{n} \in \mathrm{N}$ we have

$$
\sup _{R e z \leq(1+\varepsilon)}\left|r_{n,(1+\varepsilon)}(z)\right| \leq\left(\inf _{R e z \leq(1+\varepsilon)}\left|1-\frac{(1+\varepsilon) z}{n}\right|\right)^{-n}=\left(1-\frac{(1+\varepsilon)(1+\varepsilon)}{n}\right)^{-n}
$$

Since $(1-(1+\varepsilon)(1+\varepsilon) / \mathrm{n})^{-n} \rightarrow e^{-(1+\varepsilon)(1+\varepsilon)}$ as $\mathrm{n} \rightarrow \infty$, we have $\sup _{n}\left\|r_{n,(1+\varepsilon)}\right\|_{\infty}<\infty$. Also, by hypothesis, $\left.\sum_{j}\left\|r_{n,(1+\varepsilon)}\left(A_{j}\right)\right\|=\sum_{j} \|(1-(1+\varepsilon) / \mathrm{n}) A_{j}\right)^{-n} \|=$

$\sum_{j} \|\left[\left(n /(1+\varepsilon) R\left(n /\left((1+\varepsilon), A_{j}\right)\right]^{n} \| \leq \mathrm{M}\right.\right.$ for all $\mathrm{n} \in \mathbb{N}$.

Applying the Convergence Lemma yields $\sum_{j}\left\|e^{(1+\varepsilon) A_{j}}\right\| \leq \mathrm{M}$, as desired.

\section{Remark 3.3}

A more careful statement of the Convergence lemma and equally careful analysis of the above proof would lead to the statement that for each $\mathrm{x} \in X$. One has $\lim _{n} r_{n,(1+\varepsilon)}\left(A_{j}\right) x=e^{(1+\varepsilon) A_{j}}$ uniformly in $(1+\varepsilon)$ from compact subintervals of $[0, \infty)$.

\section{THE TROTTER-KATO THEOREM}

While in the convergence lemma the function is approximated and the sequence of operators are fixed, in the following fix the function and approximate the sequence of operators. The correct setup requires that the approximates $\left(A_{j}\right)_{n}$ are "of the same type"' as the sequence of operators, with the relevant constants being uniformly bounded. 
More precisely a family of the sequence of operators $\left(\left(A_{j}\right)_{i}\right)_{i}$ is called uniformly of half-plane type $(1+\varepsilon) \in \mathbb{R} \cup\{-\infty\}$ if each $\left(A_{j}\right)_{i}$ are of half-plane type $(1+\varepsilon)$ and $\sup _{i} M_{(\beta+\varepsilon)}\left(A_{j}\right)_{i}<\infty$ for each $\beta+\varepsilon>\infty$ (see,e.g.,[9]).

\section{Example 4.1}

Let $A_{j}$ be of half-plane type 0 , and let $\left(A_{j}\right)_{(1+\varepsilon)}:=(1+\varepsilon) A_{j} \mathrm{R}\left((1+\varepsilon), A_{j}\right)$ for $\varepsilon>0$ be the Yosida approximants. Then the family $\left(\left(A_{j}\right)_{(1+\varepsilon)}\right)_{\varepsilon>0}$ are uniformly of half-plane type 0 . Indeed, a little computation shows

$$
\sum_{j} \mathrm{R}\left(\mu^{j},\left(A_{j}\right)_{(1+\varepsilon)}\right)=\sum_{j} \frac{(1+\varepsilon)^{2}}{\left(\mu^{j}+(1+\varepsilon)\right)^{2}} R\left(\frac{(1+\varepsilon) \mu^{j}}{(1+\varepsilon)+\mu^{j}}, A_{j}\right)+\frac{1}{(1+\varepsilon)+\mu^{j}}
$$

For the second term have $\sum_{j}\left|(1+\varepsilon)+\mu^{j}\right|^{-1} \leq \min \left((1+\varepsilon)^{-1},\left(\operatorname{Re} \mu^{j}\right)^{-1}\right)$.To estimate the first compute

$$
R e \sum_{j}\left(\frac{\mu^{j}(1+\varepsilon)}{(1+\varepsilon)+\mu^{j}}\right)=\sum_{j} \frac{(1+\varepsilon)^{2} R e \mu^{j}+(1+\varepsilon)\left|\mu^{j}\right|^{2}}{\left|(1+\varepsilon)+\mu^{j}\right|^{2}}
$$

If $A_{j}$ be the sequence of generators of a bounded $C_{0}$-semigroup, it satisfies an estimate $\sum_{j}\left\|\mathrm{R}\left(\mu^{j}, A_{j}\right)\right\| \leq$ $(1+\varepsilon) \sum_{j}\left(\operatorname{Re} \mu^{j}\right)-1$ for some $\varepsilon>0$ and all $\operatorname{Re} \mu^{j}>0$. Given this, one obtains

$$
\begin{aligned}
& \sum_{j}\left\|R\left(\mu^{j},\left(A_{j}\right)_{(1+\varepsilon)}\right)\right\| \leq \sum_{j} \frac{(1+\varepsilon)^{2}}{\left|(1+\varepsilon)+\mu^{j}\right|^{2}} \frac{(1+\varepsilon) \mu^{j^{2}}}{(1+\varepsilon)^{2} R e \mu^{j}+(1+\varepsilon)\left|\mu^{j}\right|^{2}}+\sum_{j} \frac{1}{R e(1+\varepsilon)+\mu^{j}} \\
& \leq \sum_{j} \frac{(\varepsilon+2)}{\operatorname{Re} \mu^{j}}
\end{aligned}
$$

independent of $(1+\varepsilon)$. In the general case, fix $\varepsilon>-\beta$, take $\operatorname{Re} \mu^{j} \geq \beta+\varepsilon$ and define $\varepsilon:=\beta+\varepsilon /(2(\beta+\varepsilon)+1)$. It follows that $\operatorname{Re}\left(\mu^{j} /\left(2 \operatorname{Re} \mu^{j}+1\right)\right) \geq \varepsilon$ and this implies $(1+\varepsilon)^{2}\left(\operatorname{Re} \mu^{j}-\varepsilon\right) \geq 2(1+\varepsilon) \varepsilon \operatorname{Re} \mu^{j}$, since $\varepsilon>0$, From this conclude that

$$
\operatorname{Re} \sum_{j}\left(\frac{\mu^{j}(1+\varepsilon)}{(1+\varepsilon)+\mu^{j}}\right)=\sum_{j} \frac{(1+\varepsilon)^{2} R e \mu^{j}+(1+\varepsilon)\left|\mu^{j}\right|^{2}}{\left|(1+\varepsilon)+\mu^{j}\right|^{2}} \geq \varepsilon
$$

and hence that

$$
\begin{gathered}
\sum_{j}\left\|R\left(\mu^{j},\left(A_{j}\right)_{(1+\varepsilon)}\right)\right\| \leq \sum_{j} \frac{(1+\varepsilon)^{2}}{\left((1+\varepsilon)+R e \mu^{j}\right)^{2}}(1+\varepsilon)_{\varepsilon}\left(A_{j}\right)+\frac{1}{R e \mu^{j}+(1+\varepsilon)} \\
\leq \sum_{j}(1+\varepsilon)_{\varepsilon}\left(A_{j}\right)+1
\end{gathered}
$$

independent of $\varepsilon>0$ and $\mu^{j} \geq \beta+\varepsilon$.

In the previous example clearly have $\lim _{(1+\varepsilon) \rightarrow \infty} \mathrm{R}\left(\mu^{j},\left(A_{j}\right)_{(1+\varepsilon)}\right)=\mathrm{R}\left(\mu^{j}, A_{j}\right)$ in norm uniformly in $\mu^{j}$ from compact subsets of the open halfplane $(\operatorname{Re} z>0)$.

\section{Proposition 4.2}

Let $\left(\left(A_{j}\right)_{n}\right)_{n}$ be a family of sequence of operators, uniform of half-plane type with $(1+\varepsilon)_{0}\left(A_{j}\right) \leq 0$, and let $A_{j}$ be the sequence of operators such that $\mathrm{R}\left(\mu^{j},\left(A_{j}\right)_{n}\right) \rightarrow \mathrm{R}\left(\mu^{j}, A_{j}\right)$ in norm/strongly for all $\operatorname{Re} \mu^{j}>0$. Then $A_{j}$ is also of half-plane type 0 . Moreover, for $0<\varepsilon<\infty$ and $f_{j} \in \mathcal{E}\left(L_{(1+\varepsilon)}\right)$ one has $f_{j}\left(\left(A_{j}\right)_{n}\right) \rightarrow f_{j}$ $\left(A_{j}\right)$ in norm /strongly. 
Suppose furthermore that $A_{j}$ are densely defined. If $f_{j} \in H^{\infty}\left(L_{(1+\varepsilon)}\right)$ and $f_{j}\left(\left(A_{j}\right)_{n}\right) \in \mathcal{L}(\mathrm{X})$ for all $\mathrm{n} \in \mathrm{N}$ with $\mathrm{C}:=\sup _{n} \sum_{j}\left\|f_{j}\left(A_{j}\right)_{n}\right\|<\infty$, then also $f_{j}\left(A_{j}\right) \in \mathcal{L}(\mathrm{X})$, and $f_{j}\left(\left(A_{j}\right)_{n}\right) \rightarrow f_{j}\left(A_{j}\right)$ strongly.

\section{Proof}

The first two assertions are straightforward. Suppose that $A_{j}$ is densely defined; by Lemma 2.3 this implies already that $\mathcal{D}\left(A_{j}^{2}\right)$ is dense in $\mathrm{X}$. Take $\mathrm{x} \in \mathcal{D}\left(A_{j}^{2}\right), f_{j} \in H^{\infty}\left(L_{(1+\varepsilon)}\right)$ and $\mathrm{e}(\mathrm{z}):=f_{j}(\mathrm{z})(1-\mathrm{z})^{-2}$ $\in \mathcal{E}\left(L_{(1+\varepsilon)}\right)$.

Then $\sum_{j}\left\|\mathrm{e}\left(\left(A_{j}\right)_{n}\right)\left(1-A_{j}\right)^{2} \mathrm{x}\right\|=\sum_{j}\left\|f_{j}\left(\left(A_{j}\right)_{n}\right) \mathrm{R}\left(1,\left(A_{j}\right)_{n}\right)^{2}\left(1-A_{j}\right)^{2} \mathrm{x}\right\| \leq \mathrm{C} \sum_{j} \| \mathrm{R}\left(1,\left(A_{j}\right)_{n}\right)^{2}(1-$ Aj) $2 \mathrm{x}$.

Since know already that $\mathrm{e}\left(\left(A_{j}\right)_{n}\right) \rightarrow \mathrm{e}\left(A_{j}\right)$ and $\mathrm{R}\left(1,\left(A_{j}\right)_{n}\right) \rightarrow \mathrm{R}\left(1, A_{j}\right)$, conclude that

$\sum_{j}\left\|f_{j}\left(A_{j}\right) \mathrm{x}\right\|=\sum_{j}\left\|\mathrm{e}\left(A_{j}\right)\left(1-A_{j}\right)^{2} \mathrm{x}\right\| \leq \mathrm{C} \sum_{j}\left\|\mathrm{R}\left(1, A_{j}\right)^{2}\left(1-A_{j}\right)^{2} \mathrm{x}\right\|=\mathrm{C}\|\mathrm{x}\|$.

Since $\mathcal{D}\left(A_{j}^{2}\right)$ is dense, it follows that $f_{j}\left(A_{j}\right) \in \mathcal{L}(\mathrm{X})$ with $\sum_{j}\left\|f_{j}\left(A_{j}\right)\right\| \leq \mathrm{C}$. To prove that $f_{j}\left(\left(A_{j}\right)_{n}\right) \rightarrow$ $f_{j}\left(A_{j}\right)$ (strongly), need only to show $f_{j}\left(\left(A_{j}\right)_{n}\right) \mathrm{x} \rightarrow f_{j}\left(A_{j}\right) \mathrm{x}$ for all $\mathrm{x} \in \mathcal{D}\left(A_{j}^{2}\right)$. So take $\mathrm{x} \in \mathcal{D}\left(A_{j}^{2}\right)$ and let $\mathrm{y}:=$ $\left(1-A_{j}\right)^{2} \mathrm{x}$. Have seen above that $f_{j}\left(\left(A_{j}\right)_{n}\right) \mathrm{R}\left(1,\left(A_{j}\right)_{n}\right)^{2} \mathrm{y} \rightarrow f_{j}\left(A_{j}\right) \mathrm{x}$, so estimate the difference

$\sum_{j}\left\|f_{j}\left(\left(A_{j}\right)_{n}\right) \mathrm{x}-f_{j}\left(\left(A_{j}\right)_{n}\right) \mathrm{R}\left(1,\left(A_{j}\right)_{n}\right)^{2} \mathrm{y}\right\|=$

$\sum_{j}\left\|f_{j}\left(\left(A_{j}\right)_{n}\right) \mathrm{R}\left(1, A_{j}\right)^{2} \mathrm{y}-f_{j}\left(\left(A_{j}\right)_{n}\right) \mathrm{R}\left(1,\left(A_{j}\right)_{n}\right)^{2} \mathrm{y}\right\| \leq$

$\mathrm{C} \sum_{j}\left\|\mathrm{R}\left(1, A_{j}\right)^{2} \mathrm{y}-\mathrm{R}\left(1,\left(A_{j}\right)_{n}\right)^{2} \mathrm{y}\right\| \rightarrow 0$

by hypothesis.

\section{Remark 4.3}

As in the Convergence Lemma, there is version of Position 4.2 that yields some uniformity that $\left(\left(f_{j}\right)_{i}\right)_{i} \subset$ $H^{\infty}\left(L_{(1+\varepsilon)}\right)$ is uniformly bounded and $\sup _{i, n} \sum_{j}\left\|\left(f_{j}\right)_{i}\left(\left(A_{j}\right)_{n}\right)\right\|<\infty$. Then the convergence $\left.\left(f_{j}\right)_{i}\left(\left(A_{j}\right)_{n}\right) x \rightarrow\left(A_{j}\right)_{i}\left(A_{j}\right) x\right)$ is uniform in $\mathrm{i}$, for every $\mathrm{x} \in X$.

\section{Theorem 4.4}

(Trotter-Kato) Suppose that, for each $\mathrm{n} \in \mathbb{N}$, is the sequence of generators of $\mathrm{C}_{0}$-semigroup, and that $\sum_{j}\left\|e^{(1+\varepsilon)\left(A_{j}\right)_{i}}\right\| \leq \mathrm{M}$ for all $\varepsilon>-1, \mathrm{n} \in \mathbb{N}$. Suppose further that $A_{j}$ are densely defined sequence of operators and for some $\varepsilon_{0}>-1$ one has $(1+\varepsilon)_{0} \in\left(A_{j}\right)$ and $\mathrm{R}\left((1+\varepsilon)_{0},\left(A_{j}\right)_{n}\right) \rightarrow \mathrm{R}\left((1+\varepsilon)_{0}, A_{j}\right)$ strongly. Then $A_{j}$ sequence of generates $\mathrm{C}_{0}$-semigroup and one has $e^{(1+\varepsilon)\left(A_{j}\right)_{n}} \mathrm{X} \rightarrow e^{(1+\varepsilon) A_{j}} \mathrm{X}$ uniformly in $0 \leq \varepsilon \leq \infty$, for each $\mathrm{x} \in \mathrm{X}, \varepsilon>-1$.

\section{Proof}

The theorem is a consequence of Proposition 4.2 and the remark next to it, as soon as can show that actually $\{\operatorname{Re} z>o\} \subset \mathrm{e}\left(A_{j}\right)$ and $\mathrm{R}\left(\mu^{j},\left(A_{j}\right)_{n}\right) \rightarrow R\left(\mu^{j}, A_{j}\right)$ for all $\mu^{j}>0$. This is done like in [3, Proposition 111.4.]

\section{Remark 4.5}

A common assumption on $A_{j}$ implying the resolvent convergence is the following: the sequence of operators $A_{j}$ are densely defined, $\left((1+\varepsilon)_{0}-A_{j}\right)$ has dense range, and there exists a core $\mathcal{D}$ of $A_{j}$ such that $\left(A_{j}\right)_{n} \mathrm{x} \rightarrow\left(A_{j}\right) \mathrm{x}$ for all $\mathrm{x} \in \mathcal{D}$. See [3, Theorem 111.4.9].

However, one might not always be given the sequence of operators $A_{j}$. Its existence is ensured by the following condition: $\mathrm{R}\left((1+\varepsilon)_{0},\left(A_{j}\right)_{n}\right) \rightarrow \mathrm{Q} \in \mathcal{L}(\mathrm{X})$ strongly, and $\mathrm{Q}$ has dense range. Indeed, by general arguments as in [7, Appendix A.5] one has $\mathrm{Q}=\mathrm{R}\left((1+\varepsilon)_{0}, A_{j}\right)$ for some possibly multi-valued of the 
sequence of operators $A_{j}$, which is densely defined by the range assumption on $\mathrm{Q}$. It remains to show that $A_{j}$ is in fact single-valued, i.e., $\mathrm{Q}$ is injective.

\section{The Universal Model and the Phillips Calculus}

The functional calculus for the sequence of operators of half-plane type provides us with a wealth of functions $f_{j}$ where $f_{j}\left(A_{j}\right)$ are defined. It uses the Cauchy formula and only some information of the growth of the resolvent. To obtain more information, one has to make stronger assumptions, typically of the type that certain $f_{j}\left(A_{j}\right)$ are required to be bounded sequence operators. An averaging over the $\left(f_{j}^{\prime}\right) \mathrm{s}$ yields new representation formulas different from the Cauchy formula, and so more information about the functional calculus [9].

As an example of these fairly general considerations, let us suppose that $A_{j}$ the sequence of generates a bounded $C_{0}$-semigroup $\left(T^{j}(1+\varepsilon)\right)_{\varepsilon>-1}$. For a finite measure

$\mu^{j} \in \mathbf{M}[0, \infty)$ one defines its Laplace transform by

$$
\mathcal{L} \sum_{j}\left(\mu^{j}\right)(z)=\int e^{z(1+\varepsilon)} \sum_{j} \mu^{j}(d(1+\varepsilon))(\operatorname{Re} z \leq 0)
$$

It is well-known that $\mathcal{L}\left(\mu^{j}\right)$ is holomorphic on $(\operatorname{Re} \mathrm{z}<0)$, and bounded and continuous on $(\operatorname{Re} \mathrm{z} \leq 0)$. Moreover, $\mu^{j}$ is uniquely determined by $\mathcal{L}\left(\mu^{j}\right)$.

If $f_{j}=\mathcal{L}\left(\mu^{j}\right)$ we define

$$
\sum_{j} f_{j}\left(A_{j}\right) x=\int_{[0, \infty)} \sum_{j} T^{j}((1+\varepsilon)) \mu^{j}(d(1+\varepsilon))(x \in X)
$$

This yields an algebra homomorphism $\left(\mu^{j} \rightarrow \mathcal{L}\left(\mu^{j}\right)\left(A_{j}\right)\right): \mathbf{M}[0, \infty) \rightarrow \mathcal{L}(\mathrm{X})$.

By the following lemma, this is in accordance with the previous definitions.

\section{Lemma 5.1}

Let $\varepsilon>-1$, and let $f_{j} \in \mathcal{E}\left(L_{(1+\varepsilon)}\right)$. Then there is functionsg ${ }^{j} \in \boldsymbol{L}^{\mathbf{1}}(0, \infty)$ such that

$$
\sum_{j} f_{j}\left(A_{j}\right)=\int_{0}^{\infty} \sum_{j} \mathrm{~g}^{j}(1+\varepsilon) T^{j}(1+\varepsilon) d(1+\varepsilon)
$$

If $f_{j} \in H^{\infty}\left(L_{(1+\varepsilon)}\right)$ is such that there is $\mu^{j} \in \mathbf{M}[0, \infty)$ with $f_{j}=\mathcal{L}\left(\mu^{j}\right)$, then indeed

$$
\sum_{j} f_{j}\left(A_{j}\right)=\int_{[0, \infty]} \sum_{j} T^{j}(1+\varepsilon) \mu^{j}(d(1+\varepsilon))
$$

Where the left-hand side is defined by the functional calculus for $A_{j}$ as sequence of operators of halfplane type 0 .

Proof

To prove the first statement let $(1+\varepsilon) \in(0,(1+\varepsilon))$ and define

$\sum \mathrm{g}^{j}(1+\varepsilon)=\frac{1}{2 \pi i} \int_{R e z=(1+\varepsilon)} \sum_{j} f_{j}(z) e^{-z(1+\varepsilon)} d z$. Then $\mathrm{g}^{j} \in \boldsymbol{L}^{\mathbf{1}}(0, \infty)$ and Fubini's theorem does the rest.

For the second assertion assume without restriction that $\varepsilon<-1$. Define $\mathrm{e}(\mathrm{z})=(1-\mathrm{z})^{-2}$, so e $f_{j}$, e $\in \mathcal{E}$. Find $\mathrm{g}^{j} \in \boldsymbol{L}^{\mathbf{1}}(0, \infty)$ such that $\mathcal{L}\left(\mathrm{g}^{j}\right)=$ e. Then e $f_{j}=\mathcal{L}\left(\mu^{j} * \mathrm{~g}^{j}\right)$, and so 


$$
\begin{gathered}
\sum_{j}\left(e f_{j}\right)\left(A_{j}\right)=\int_{[0, \infty)} \sum T^{j}(1+\varepsilon)\left(\mu^{j} * \mathrm{~g}^{j}\right)(d(1+\varepsilon))= \\
\int_{[0, \infty)} \int_{[0, \infty)} \sum_{j} T^{j}(2(1+\varepsilon)) \mu^{j}(d(1+\varepsilon)) \mathrm{g}^{j}(1+\varepsilon) d(1+\varepsilon)=\int_{[0, \infty)} \sum_{j} T^{j}(1+\varepsilon) \mathrm{g}^{j}(1+\varepsilon) d(1+\varepsilon) \\
\int_{[0, \infty)} \sum_{j} T^{j}(1+\varepsilon) \mu^{j}(d(1+\varepsilon))=\sum_{j} e\left(A_{j}\right) \int_{[0, \infty)} T^{j}(1+\varepsilon) \mu^{j}(d(1+\varepsilon))
\end{gathered}
$$

This yields the assertion.

Important about the Phillips calculus is the fact that it is all that one can expect in general from the functional calculus for semigroup sequence of generators, introduce a very special semigroup

Example 5.2

(Shift Semigroup) Let $\mathrm{X}=\boldsymbol{L}^{\mathbf{1}}(0, \infty)$ and let $T^{j}(1+\varepsilon) f_{j}(1+\varepsilon):=f_{j}((1+\varepsilon)+(1+\varepsilon))$ for $\varepsilon>-1$. Then $T^{j}$ is as trongly continuous contraction semigroup on $\mathrm{X}$ with sequence of generators $A_{j}=$ $d / d(1+\varepsilon)$ on $\mathcal{D}\left(A_{j}\right)=\boldsymbol{W}^{\mathbf{1}, \mathbf{1}}[0, \infty)$. If we think of $\boldsymbol{L}^{\mathbf{1}}(0, \infty)$ as sitting inside $\boldsymbol{L}^{\mathbf{1}}(\mathbb{R})$, with all functions in $\boldsymbol{L}^{\mathbf{1}}(0, \infty)$ being zero on $(-\infty, 0)$, then the resolvent of $A_{j}$ can be written as $\mathrm{R}\left((1+\varepsilon), A_{j}\right) f_{j}=e_{(1+\varepsilon)} * f_{j}$ $\left.\right|_{(0, \infty)}$, with $e_{(1+\varepsilon)}(1+\varepsilon)=e^{(1+\varepsilon)(1+\varepsilon)} \mathbf{1}_{(-\infty, 0)}$, for Re $\varepsilon>-1$. It can be shown that actually $\sigma\left(A_{j}\right)=(\operatorname{Re} \mathrm{z}$ $\leq 0)$. The Phillips calculus for this particular semigroup reads

$\boldsymbol{L}\left(\mu^{j}\right)\left(A_{j}\right) f_{j}=\left(\left(\mu^{j}\right)^{\sim} * f_{j}\right) \mathbf{1}_{(0, \infty)},\left(f_{j} \in \boldsymbol{L}^{\mathbf{1}}(0, \infty)\right)$

where $\left(\mu^{j}\right)^{\sim}\left(B_{j}\right)=\mu^{j}\left(-B_{j}\right)$ for every Borel set $B_{j} \subset \mathbb{R}$.

Claim: The mapping $\left.\left(\mu^{j} \mapsto \mathcal{L}\left(\mu^{j}\right) A_{j}\right)\right): \mathbf{M}[0, \infty) \rightarrow \mathcal{L}\left(\boldsymbol{L}^{\mathbf{1}}(0, \infty)\right)$ is isometric.

Proof

Clearly the mapping is contractive. Fix $\mu^{j} \in \mathbf{M}[0, \infty)$ and denote $f_{j}:=\mathcal{L}\left(\mu^{j}\right)$. Fix $\psi_{j} \in \boldsymbol{L}^{\mathbf{1}}(0, \infty)$ and $\varphi_{j} \in \boldsymbol{C}_{\mathbf{0}}$ $[0, \infty)$ such that $\sum_{j}\left\|\psi_{j}\right\|_{1}\left\|\varphi_{j}\right\|_{\infty} \leq \infty$. Then

$$
\begin{aligned}
\sum_{j}\left|\left\langle\left(f_{j} A_{j}\right) \psi_{j}, \varphi_{j}\right\rangle\right| & =\sum_{j}\left|\int_{0}^{\infty} \psi_{j}(2(1+\varepsilon)) \mu^{j}(d(1+\varepsilon)) \varphi_{j}(1+\varepsilon) d(1+\varepsilon)\right| \\
= & \sum_{j}\left|\int_{0}^{\infty} \psi_{j}(1+\varepsilon) \int_{0}^{(1+\varepsilon)} \psi_{j}((1+\varepsilon)-(1+\varepsilon)) \mu^{j}(d(1+\varepsilon)) d(1+\varepsilon)\right|
\end{aligned}
$$

Taking the supremum with respect to $\psi_{j}$ yields

$$
\sum_{j} \sup _{\psi_{j}} \mid\left\langle\left(f_{j}\left(A_{j}\right) \psi_{j}, \varphi_{j}\right\rangle\left|=\sup _{\varepsilon>-1} \sum_{j}\right| \int_{0}^{t} \psi_{j}((1+\varepsilon)-(1+\varepsilon)) \mu^{j}(d(1+\varepsilon))\right|
$$

By choosing suitable $\varphi_{j}$ we see that

$\sum_{j} \|\left(f_{j}\left(A_{j}\right) \|=\sum_{j} \sup _{\varphi_{j}} \sup _{\psi_{j}} \mid\left\langle\left(f_{j}\left(A_{j}\right) \psi_{j}, \varphi_{j}\right\rangle|\geq| \mu^{j}\right|\left(\left[0,(1+\varepsilon)_{0}\right]\right)\right.$

for any $\varepsilon>-1$. Letting $(1+\varepsilon)_{0} \rightarrow \infty$ concludes the proof.

As a consequence one obtains the following "transference" type result [9].

\section{Corollary 5.3}

Let $A_{j}$ the sequence of generates bounded $C_{0}$-semigroup on the Banach space $\mathrm{X}$.Then 


$$
\sum_{j}\left\|f_{j}\left(A_{j}\right)\right\|_{\mathcal{L}\left(A_{j}\right)} \leq \sup _{\varepsilon>-1} \sum_{j}\left\|T^{j}(1+\varepsilon)\right\| \sum_{j}\left\|f_{j}(d / d(1+\varepsilon))\right\|_{\mathcal{L}\left(L^{1}(0, \infty)\right.}
$$

for every $f_{j} \in\left\{\boldsymbol{L}\left(\mu^{j}\right) \mid \mu^{j} \in \mathbf{M}[0, \infty)\right\}$.

\section{Proof}

Let $f_{j}=\mathcal{L}\left(\mu^{j}\right)$. Then $f_{j}\left(A_{j}\right) \mathrm{x}=\int \sum_{j} T^{j}(1+\varepsilon) x \mu^{j}(d(1+\varepsilon))$

for all $\mathrm{x} \in \mathrm{X}$, whence

$$
\sum_{j}\left\|f_{j}\left(A_{j}\right)\right\| \leq\left[\sup _{\varepsilon>-1} \sum_{j}\left\|T^{j}(1+\varepsilon)\right\|\right]\left\|\mu^{j}\right\|_{M[0, \infty)}
$$

But $\sum_{j}\left\|\mu^{j}\right\|_{M[0, \infty)}=\sum_{j}\left\|f_{j}(d / d(1+\varepsilon))\right\|$ was shown above.

We can now state the main result.

\section{Proposition 5.4}

Let $0<\varepsilon<\infty$ and let $f_{j} \in H^{\infty}\left(H_{(1+\varepsilon)}\right)$. Then the following statements are equivalent:

(i) $f_{j}\left(A_{j}\right)$ are bounded sequence operators, for each sequence of generators of a bounded semigroup $T^{j}$ on a Banach space $X$.

(ii) $f_{j}\left(A_{j}\right)$ is a bounded operator, where $A_{j}=d / d(1+\varepsilon)$ sequence generates the left shift semigroup on $\mathrm{X}$ $=L^{1}(0, \infty)$.

(iii) There exists $\mu^{j} \in \mathbf{M}[0, \infty)$ such that $f_{j}=\mathcal{L}\left(\mu^{j}\right)$.

\section{Proof}

The implications (iii) $\Rightarrow$ (i) $\Rightarrow$ (ii) are trivial. Suppose that $f_{j}\left(A_{j}\right)$ are abounded sequence operators on $\mathrm{X}=$ $\boldsymbol{L}^{\mathbf{1}}(0, \infty)$, where $A_{j}=d / d(1+\varepsilon)$ is the sequence of generators of the left shift semigroup $\left(T^{j}(1+\right.$ $\varepsilon) \mathcal{E}>-1$. Let $\left(f_{j}\right) n(\mathrm{z}):=f(\mathrm{z})[\mathrm{n} /(\mathrm{n}-\mathrm{z})]^{2}$. Then $\left(f_{j}\right) n(A j) \rightarrow f j(A j)$ strongly. By Lemma 5.1, $\left(f_{j}\right)_{n}=\mathcal{L}\left(\mathrm{g}^{j}\right)_{n}$ for certain $\left(\mathrm{g}^{j}\right)_{n} \in \boldsymbol{L}^{\mathbf{1}}(0, \infty)$. By example 5.2, $\sum_{j}\left\|\left(\mathrm{~g}^{j}\right)_{n}\right\|_{1}=\sum_{j}\left\|\left(f_{j}\right)_{n}\left(A_{j}\right)\right\|$, and this is clearly bounded independent of $n$. Since $\mathbf{C}_{0}[0, \infty)$ is separable, the weak ${ }^{*}$ topology on bounded sets of $\mathbf{M}[0, \infty)$ is metrizable. By compactness, there exists subsequence $\left(n_{k}\right)_{k}$ and a measure $\mu^{j} \in \mathbf{M}[0, \infty)$ such that $\left(\left(\mathrm{g}^{j}\right)_{n}\right)_{k} \rightarrow \mu^{j}$ weakly *. For fixed $\operatorname{Re} \mathrm{z}<0$, the function

$(1+\varepsilon) \rightarrow e^{\left(\mu^{j}\right) z}$ is in $\boldsymbol{C}_{\mathbf{0}}[0, \infty)$, and hence

$$
\sum_{j} f_{j}(z)\left[n_{k} /\left(n_{k}-z\right)\right]^{2}=\sum_{j}\left(\left(f_{j}\right)_{n}\right)_{k}(z)=\int_{0}^{\infty} \sum_{j}\left(\left(\mathrm{~g}^{j}\right)_{n}\right)_{k}(1+\varepsilon) e^{(1+\varepsilon) z} \mu^{j}(d(1+\varepsilon))
$$

But clearly $\left(\left(f_{j}\right)_{n}\right)_{k}(\mathrm{z}) \rightarrow f_{j}(\mathrm{z})$ also, whence $f_{j}=\mathcal{L}\left(\mu^{j}\right)$.

The proposition explains the importance of the Phillips calculus for results about approximation in norm, like the Post-Widder, the Phragmen-Doetschand the Complex inversion formulas, see [3, Section III.5]. In fact it shows that the proofs given there are natural.

\section{SECTORIAL AND STRONG STRIP-TyPE OPERATORS}

If the semigroup is a group, the spectrum of the sequence generators are contained in a vertical strip. So the appropriate functional calculus is for functions living on that strip and not in a half plane. For reasons that will become clear later, it is desirable to consider horizontal strips instead of vertical ones (see, e.g., [9]). 
For $\varepsilon>-1$ we define

$S(1+\varepsilon)_{(1+\varepsilon)}:=\{\mathrm{z} \in \mathbb{C}|| \operatorname{Im~} \mathrm{z} \mid<(1+\varepsilon)\}$ and $\mathrm{S}(1+\varepsilon)_{0}:=\mathbb{R}$.

\section{Definition 6.1}

The sequence of operators $A_{j}$ on a Banach space $\mathrm{X}$ is called to be of strong strip-type $\varepsilon \geq-1$ (in short: $\left.A_{j} \in \operatorname{SStrip}(1+\varepsilon)\right)$, if $\sigma\left(A_{j}\right) \subset \overline{S(1+\varepsilon)_{(1+\varepsilon)}}$ and for every $\left(\frac{1+\varepsilon}{\varepsilon}\right)>(1+\varepsilon)$ there is $M_{\left(\frac{1+\varepsilon}{\varepsilon}\right)}$ such that

$$
\sum_{j}\left\|R\left(1+\varepsilon, A_{j}\right)\right\| \leq \sum_{j} \frac{(1+\varepsilon)\left(\frac{1+\varepsilon}{\varepsilon}\right)}{|\operatorname{Im}(1+\varepsilon)|-\left(\frac{1+\varepsilon}{\varepsilon}\right)}\left(|\operatorname{Im}(1+\varepsilon)|>\left(\frac{1+\varepsilon}{\varepsilon}\right)\right)
$$

The least of the $(1+\varepsilon)$ such that $A_{j} \in \operatorname{SStrip}(1+\varepsilon)$ is denoted by $(1+\varepsilon)_{s s t}\left(A_{j}\right)$.

For example, if $-\mathrm{i} A_{j}$ sequence of generates group $(\mathrm{U}(1+\varepsilon))_{(1+\varepsilon) \in \mathbb{R}}$ such that $\|\mathrm{U}(1+\varepsilon)\| \leq \mathrm{M} e^{(1+\varepsilon)|1+\varepsilon|}$ then $A_{j}$ is of strong strip-type $(1+\varepsilon)$. One can weaken the hypothesis; in fact it suffices that the exponential group type $\theta(U) \leq(1+\varepsilon)$.

Here $\theta(\mathrm{U}):=\inf \left\{\varepsilon>-1 \mid \exists \varepsilon>0:\|\mathrm{U}(1+\varepsilon)\| \leq(1+\varepsilon) e^{(1+\varepsilon)|1+\varepsilon|}((1+\varepsilon) \in \mathbb{R})\right\}$.

With a strong strip-type the sequence of operators $A_{j} \in \operatorname{SStrip}(1+\varepsilon)$ there comes along a natural holomorphic functional calculus. One considers functions $f_{j}$ holomorphic on strips $(1+\varepsilon)(1+\varepsilon)_{\varphi_{j}}$ with $\varphi_{j}>(1+\varepsilon)$. If $\mathrm{f}$ has integrable decay a $(1+\varepsilon) \pm \infty$, e.g., $f_{j}=\mathrm{O}\left(|z|^{-(2+\varepsilon)}\right)$ as $|z| \rightarrow \infty$

some $\varepsilon>-1$, then $f_{j}\left(A_{j}\right)$ are defined by the usual Cauchy integral, the contour being the boundary of an appropriate strip. This gives a primary functional calculus for $A_{j}$ and is extended to a meromorphic functional calculus via the usual regularization procedure, see [7, Section 4.2]. Of course this functional calculus extends the two half-plane calculi available for $A_{j}$. (Note that $\pm \mathrm{i} A_{j}$ are both of halfplane-type and so, have natural functional calculi on left halfplanes.)

It is clear that a result analogous to Proposition 2.4 holds. The sequence of operators $-\mathrm{i} A_{j}$ sequence generates $C_{0}$-group if and only if $A_{j}$ is densely defined, of strong strip-type, and $\left(e^{i(1+\varepsilon) z}\right)\left(A_{j}\right)$ is bounded for all $(1+\varepsilon) \in \mathbb{R}$. In this case, one can set up a Phillips calculus and obtains

$$
\sum_{j} f_{j}\left(A_{j}\right) x=\int \sum_{j} U(1+\varepsilon) x \mu^{j}(d(1+\varepsilon))(x \in X)
$$

if $f_{j}(\mathrm{z})=\widehat{\mu^{\mathrm{j}}}(\mathrm{z})=e^{i(1+\varepsilon) z} \mu^{j}(\mathrm{~d}(1+\varepsilon))$ and $\mu^{j}$ is such that $e^{(1+\varepsilon)|1+\varepsilon|} \mu^{j}(\mathrm{~d}(1+\varepsilon)) \in \mathbf{M}(\mathbb{R})$ for some $(1+\varepsilon)>\theta(U)$. See $[6]$ for proofs.

Strong strip-type operators arise naturally as logarithms of sectorial operators [7, Proposition 3.5.1], and the fact that there are sectorial sequence of operators without bounded imaginary powers yields the existence of natural examples of strong strip-type sequence of operators that do not sequence of generates group. For the sake of completeness, give the definition of sectorial sequence of operators. Let $(1+\varepsilon) \in$ $[0, \pi]$ and define

$$
S_{(1+\varepsilon)}=\left\{e^{z} \mid z \in S(1+\varepsilon)_{(1+\varepsilon)}\right\}\left\{\begin{array}{c}
z \neq 0 \mid \arg z<(1+\varepsilon),(\varepsilon>-1) \\
{[0, \infty),(\varepsilon=-1)}
\end{array}\right.
$$

\section{Definition 6.2}

The sequence of operators $A_{j}$ on a Banach space $\mathrm{X}$ is called sectorial of angle $(1+\varepsilon)<\pi$ if $\sigma\left(A_{j}\right)$ $\subset \overline{S_{(1+\varepsilon)}}$ and for every $\left(\frac{1+\varepsilon}{\varepsilon}\right) \in((1+\varepsilon), \pi]$ there is $M_{\left(\frac{1+\varepsilon}{\varepsilon}\right)}$ such that

$\sum_{j}\left\|(1+\varepsilon) \mathrm{R}\left((1+\varepsilon), A_{j}\right)\right\| \leq M_{\left(\frac{1+\varepsilon}{\varepsilon}\right)}\left(|\arg (1+\varepsilon)| \in\left[\left(\frac{1+\varepsilon}{\varepsilon}\right), \pi\right]\right)$. 
The least $(1+\varepsilon)$ such that $A_{j} \in \operatorname{Sect}(1+\varepsilon)$, is denoted by $(1+\varepsilon)_{s e c}\left(A_{j}\right)$.

For sectorial operators there is a natural meromorphic functional calculus set up exactly in the same way as for the sequence of operators of strip- or of halfplane-type [7].(The functions live on larger sectors $S_{\varphi_{j}}$, of course). The pay-off for semigroup theory lies in the fact that sequence of operators $-A_{j}$ generates a bounded holomorphic semigroup if and only if $A_{j}$ is sectorial of type < $\pi / 2$. [7, Section 3.4].

There is strong link between sectorial and strip-type operators. It was proved essentially by Nollau that if $A_{j}$ is an injective sectorial sequence of operators of angle $(1+\varepsilon)$ then $\log A_{j}$ is of strong strip-type $(1+\varepsilon)$. In fact, it was proved in [4] that the strip-type of $\log \left(A_{j}\right)$ is equal to the sectoriality type of $A_{j}:(1+\varepsilon)_{s s t}$ $\left(\log \left(A_{j}\right)\right)=(1+\varepsilon)_{s e c}\left(A_{j}\right)$. In [5] could even show that the spectral mapping theorem $\sigma\left(\log \left(A_{j}\right)\right)=$ $\log \left(\sigma\left(A_{j}\right) \backslash\{0\}\right.$ holds.

One can switch back and forth from $A_{j}$ to $\log A_{j}$, as far as the functional calculi are concerned. Indeed, there is a composition rule $f_{j}\left(\log A_{j}\right)=\left(f_{j}(\log \mathrm{z})\right)\left(A_{j}\right)$ in the sense that $f_{j}\left(\log \left(A_{j}\right)\right)$ is defined if and only if $\left(f_{j}(\log \mathrm{z})\right)\left(A_{j}\right)$ is defined. The sequence of operators $-\mathrm{i} \log \left(A_{j}\right)$ sequence generates $C_{0}$-group if and only if $A_{j}$ has bounded imaginary powers $\left(A_{j}^{i(1+\varepsilon)}\right)_{(1+\varepsilon) \in \mathbb{R}}$. (Since $e^{i(1+\varepsilon) \log z}=z^{i(1+\varepsilon)}$ and in view of the composition rule above, this does not come as a surprise.) See [7] for proofs and more information. The symmetry between (injective) sectorial and strip-type sequence of operators, however, is only partial. There are strong strip-type operators that are not logarithms of sectorial ones. (An example is $-d / \mathrm{d}(1+\varepsilon)$ on $\left.\boldsymbol{L}^{\mathbf{1}}(\mathbb{R})\right)$. Here, the best result up to now isbyMonniaux [8]; it states that if $-\mathrm{i} A_{j}$ sequence generates a $C_{0}$-group $\mathrm{U}$ of type $\theta(\mathrm{U})<\pi$, and if the space $\mathrm{X}$ is $\mathrm{UMD}$, then $A_{j}$ is the logarithm of a sectorial sequence of operators. See [6, Section 2.6 and 5.1] for a recent new proof.

Let us note that as in the semi group case, there are Convergence Lemmas and Trotter- Kato type results for sectorial and strip-type sequence of operators, cp. [7, Section 2.6 and 5.1]

\section{COSINE Functions}

Turn to the treatment by functional calculus methods of cosine the sequence of operators functions. As a guiding intuition, think of the sequence of generators $-A_{j}$ of a cosine function as $-A_{j}=\left(-\mathrm{i} B_{j}\right)^{2}$ where $\mathrm{i}\left(B_{j}\right)$ the sequence of generate generates group [9] . It is then pretty natural to consider a functional calculus on the parabola $\Pi_{(1+\varepsilon)}=\left\{\mathrm{z}^{2} \mid \mathrm{z} \in \mathrm{S}(1+\varepsilon)_{(1+\varepsilon)}\right\}$.

To define an operator of parabola-type $(1+\varepsilon)$ need to specify a resolvent estimate that should hold outside every larger parabola. A natural way to find such a condition is to look at the negative sequence of generators of cosine functions.

So let $-A_{j}$ sequence of generates a cosine function Cos on the Banach space $\mathrm{X}$, and assume that

$$
\begin{aligned}
& \|\operatorname{Cos}(1+\varepsilon)\| \leq \mathrm{M} e^{(1+\varepsilon)|1+\varepsilon|},(1+\varepsilon) \in \mathbb{R} \text {. By definition, } \\
& \quad(1+\varepsilon) R \sum_{j}\left((1+\varepsilon)^{2},-A_{j}\right)=\int_{0}^{\infty} e^{-(1+\varepsilon)(1+\varepsilon)} \operatorname{Cos}(1+\varepsilon) d(1+\varepsilon)(\operatorname{Re}(1+\varepsilon)>(1+\varepsilon))
\end{aligned}
$$

Taking norms and estimating yields

$$
\sum_{j}\left\|R\left(-(1+\varepsilon)^{2}, A_{j}\right)\right\| \leq \frac{M}{|1+\varepsilon|(R e(1+\varepsilon)-(1+\varepsilon))}(R e(1+\varepsilon)>(1+\varepsilon))
$$

The function $\left(\mathrm{z} \rightarrow \mathrm{z}^{2}\right):(\operatorname{Im~} \mathrm{z}>(1+\varepsilon)) \rightarrow \mathbb{C} / \overline{\pi_{(1+\varepsilon)}}$ is biholomorphic, its inverse being on a branch of the square root. Writing $\mu^{j}=-(1+\varepsilon)^{2}$ in (1) yields

$$
\sum_{j}\left\|R\left(\mu^{j}, A_{j}\right)\right\| \leq M \sum \frac{1}{\left.\sqrt{\left|\mu^{j}\right|}\left(\left|\operatorname{Im} \sqrt{\mu^{j}}\right|\right)-(1+\varepsilon)\right)}\left(\mu^{j} \notin \overline{\Pi_{(1+\varepsilon)}}\right)
$$


This expression is actually independent of the branch of the square root take. It yields the canonical resolvent estimate for a parabola-type sequence of operators.

\section{Definition 7.1}

Let $\varepsilon>-1$ and define $\Pi_{(1+\varepsilon)}:=\left\{\mathrm{z}^{2} \mid \mathrm{z} \in \mathrm{S}(1+\varepsilon)_{(1+\varepsilon)}\right\}$. The sequence of operators $A_{j}$ on a Banach space $\mathrm{X}$ is called to be of parabola-type $(1+\varepsilon)$ (in short: $\left.A_{j} \in \operatorname{Para}(1+\varepsilon)\right)$ if $\sigma\left(A_{j}\right) \subset \overline{\Pi_{(1+\varepsilon)}}$ and for each $\left(\frac{1+\varepsilon}{\varepsilon}\right)>(1+\varepsilon)$ there exists $M_{\left(\frac{1+\varepsilon}{\varepsilon}\right)}$ such that

$$
\sum_{j}\left\|R\left(\mu^{j}, A_{j}\right)\right\| \leq \sum_{j} \frac{M_{\left(\frac{1+\varepsilon}{\varepsilon}\right)}}{\left.\sqrt{\left|\mu_{j}\right|}\left(\mid \operatorname{Im} \sqrt{\mu^{j} \mid}\right)-\left(\frac{1+\varepsilon}{\varepsilon}\right)\right)}\left(\mu_{j} \notin \overline{\Pi_{\left(\frac{1+\varepsilon}{\varepsilon}\right)}}\right)
$$

The least of the $(1+\varepsilon)$ such that $A_{j} \in \operatorname{Para}(1+\varepsilon)$ is denoted by $(1+\varepsilon)_{\text {par }}\left(A_{j}\right)$.

Have seen above that if $-A_{j}$ sequence of generates a cosine function of exponential growth type $(1+\varepsilon)$ then $A_{j}$ is of parabola-type $(1+\varepsilon)$. Here is another example.

\section{Lemma 7.2}

Let $B_{j} \in \operatorname{SStrip}(1+\varepsilon)$. Then $A_{j}:=\left(B_{j}\right)^{2} \in \operatorname{Para}(1+\varepsilon)$.

Proof

Fix $\left(\frac{1+\varepsilon}{\varepsilon}\right)>(1+\varepsilon)$ and $\mu^{j} \in \mathbb{C} / \overline{\Pi_{\left(\frac{1+\varepsilon}{\varepsilon}\right)}}$. Since $B_{j}$ is of strong strip-type, find $M_{\left(\frac{1+\varepsilon}{\varepsilon}\right)}$ such that

$\sum_{j}\left\|\mathrm{R}\left((1+\varepsilon), B_{j}\right)\right\| \leq M_{\left(\frac{1+\varepsilon}{\varepsilon}\right)}\left(|\operatorname{Im}(1+\varepsilon)|-\left(\frac{1+\varepsilon}{\varepsilon}\right)\right)^{-1}\left(|\operatorname{Im}(1+\varepsilon)|>\left(\frac{1+\varepsilon}{\varepsilon}\right)\right)$.

Taking $(1+\varepsilon):=\sqrt{\mu^{j}}$ (either choice) yields.

$$
\begin{gathered}
R\left(\mu^{j}, A_{j}\right)=\left((1+\varepsilon)^{2}-\left(B_{j}\right)^{2}\right)^{-1}=-R\left((1+\varepsilon), B_{j}\right) R\left(-(1+\varepsilon), B_{j}\right) \\
=\frac{1}{2(1+\varepsilon)}\left(R\left((1+\varepsilon), B_{j}\right)-R\left(-(1+\varepsilon), B_{j}\right)\right)
\end{gathered}
$$

by the resolvent identity. Estimating the norm yields the assertion.

And yet another one.

\section{Lemma 7.3}

Let $\varepsilon>-1$ and let $A_{j}$ be sequence of operators on a Banach space X such that $\sigma\left(A_{j}\right) \subset \Pi_{(1+\varepsilon)}$ and $\sum_{j}\left\|\mathrm{R}\left(\mu^{j}, A_{j}\right)\right\| \leq(1+\varepsilon) \operatorname{dist}\left(\mu^{j}, \Pi_{(1+\varepsilon)}\right)^{-1}\left(\mu^{j} \notin \overline{\Pi_{(1+\varepsilon)}}\right)$ for some $\varepsilon>0$. Then $A_{j} \in \operatorname{Para}(1+\varepsilon)$. More precisely,

$$
\sum_{j}\left\|R\left(\mu^{j}, A_{j}\right)\right\| \leq \sum_{j} \frac{(1+\varepsilon)}{\left.\sqrt{\left|\mu^{j}\right|}\left(\left|\operatorname{Im} \sqrt{\mu^{j}}\right|\right)-(1+\varepsilon)\right)}\left(\mu^{j} \notin \overline{\Pi_{(1+\varepsilon)}}\right)
$$

\section{Proof}

Let $|\operatorname{Im}(1+\varepsilon)|>(1+\varepsilon)$. It suffices to show that

$|(1+\varepsilon)|(|\operatorname{Im}(1+\varepsilon)|-(1+\varepsilon)) \leq \operatorname{dist}\left((1+\varepsilon)^{2}, \Pi_{(1+\varepsilon)}\right)$, i.e.

$\left|(1+\varepsilon)^{2}-z^{2}\right|=|(1+\varepsilon)-\mathrm{z}||(1+\varepsilon)+\mathrm{z}| \leq|(1+\varepsilon)|(|\operatorname{Im}(1+\varepsilon)|-(1+\varepsilon))$

for all $\mathrm{z}$ such that $|\operatorname{Im} \mathrm{z}| \leq(1+\varepsilon)$. But $|\mathrm{z}-(1+\varepsilon)| \geq(|\operatorname{Im}(1+\varepsilon)|-(1+\varepsilon))$ and $|\mathrm{z}+(1+\varepsilon)| \geq$ $(|\operatorname{Im}(1+\varepsilon)|-(1+\varepsilon))$. And at least one of the factors $|(1+\varepsilon)-\mathrm{z}|,|(1+\varepsilon)+\mathrm{z}| \mathrm{i}(1+\varepsilon) \geq$ $|(1+\varepsilon)|$, for trivial geometrical reasons. 


\section{Corollary 7.4}

Let $\mathrm{X}=\mathrm{H}$ be a Hilbert space and let $A_{j}$ be such the condition of lemma7.3 with $\varepsilon=0$, and so is of parabola-type. That its numerical range and spectrum is contained in $\overline{\Pi_{(1+\varepsilon)}}$. Then $A_{j}$ satisfies with $\varepsilon=0$, and so is of parabola-type.

Set up a functional calculus for a parabola-type of the sequence of operators $A_{j} \in \operatorname{Para}(1+\varepsilon)$ in the obvious fashion. Let $\varphi_{j}>(1+\varepsilon)$ and let $\mathcal{E}\left(\Pi_{\varphi_{j}}\right):=f_{j} \in\left\{\mathcal{O}\left(\Pi_{\varphi_{j}}\right) \mid f_{j}(\mathrm{z})=\mathrm{O}\left(|z|^{-\mathcal{E}}\right)\right.$ as $\mathrm{z} \rightarrow \infty$,

for some $\varepsilon>1 / 2$ \} Then

$$
f_{j}(1+\varepsilon)=\frac{1}{2 \pi i} \int_{\partial \Pi_{\left(\frac{1+\varepsilon}{\varepsilon}\right)}} \sum_{j} f_{j}(z) \frac{d z}{z-(1+\varepsilon)}\left((1+\varepsilon) \in \Pi_{\left(\frac{1+\varepsilon}{\varepsilon}\right)}\right)
$$

by Cauchy's theorem, where $\left(\frac{1+\varepsilon}{\varepsilon}\right) \in\left((1+\varepsilon), \varphi_{j}\right)$. Define

$$
f_{j}\left(A_{j}\right)=\frac{1}{2 \pi i} \int_{\partial \Pi_{\left(\frac{1+\varepsilon}{\varepsilon}\right)}} \sum_{j} f_{j}(z) R\left(z, A_{j}\right) d z=\frac{-1}{\pi i} \int_{i\left(\frac{1+\varepsilon}{\varepsilon}\right)-\infty}^{i\left(\frac{1+\varepsilon}{\varepsilon}\right)+\infty} f_{j}\left(z^{2}\right) z R\left(z^{2}, A_{j}\right) d z
$$

and as usual this does not depend on the choice of $\left(\frac{1+\varepsilon}{\varepsilon}\right)$. In the usual fashion one shows that this defines an algebra homomorphism $\Phi:=\left(f_{j} \rightarrow f_{j}\left(A_{j}\right)\right): \mathcal{E}\left(\Pi_{\varphi_{j}}\right) \rightarrow \mathcal{L}(\mathrm{X})$, and that it respects resolvents:

$((1+\varepsilon)-\mathrm{z})^{-1}\left(A_{j}\right)=\mathrm{R}\left((1+\varepsilon), A_{j}\right)\left((1+\varepsilon) \notin \Pi_{\varphi_{j}}\right)$.

Therefore, a meromorphic functional calculus in the sense of [7, Section 1.3] is defined, and there is a canonical definition of $f_{j}\left(A_{j}\right)$ for meromorphic functions $\mathrm{f}$ on $\Pi_{\varphi_{j}}$ that are regular is able by elements of $\mathcal{E}\left(\Pi_{\varphi_{j}}\right)$. Of course one obtains a corresponding Convergence Lemma in the case that $A_{j}$ is densely defined.

If the parabola-type of the sequence operators $A_{j}$ arises as a square $A_{j}=\left(B_{j}\right)^{2}$ of a strong strip-type sequence of operators $B_{j}$, then there is an obvious composition rule:

\section{Proposition 7.5}

Let $B_{j} \in \operatorname{SStrip}(1+\varepsilon)$ and $A_{j}:=\left(B_{j}\right)^{2}$. Let $\varphi_{j}>(1+\varepsilon)$ and $f_{j} \in \mathcal{M}\left(\Pi_{\varphi_{j}}\right)$ such that $f_{j}\left(A_{j}\right)$ is defined. Then $\left[f_{j}\left(\mathrm{z}^{2}\right)\right]\left(B_{j}\right)$ is defined and $f_{j}\left(\mathrm{z}^{2}\right)\left(B_{j}\right)=f_{j}\left(A_{j}\right)$.

Proof.

By [7, Proposition 1.3.6] one may suppose without loss of generality that $f_{j} \in \mathcal{E}\left(\Pi_{\varphi_{j}}\right)$. Then one may perform a computation similar to [7, p.43,p.96/97]. Butit is even simpler:

$$
\begin{gathered}
\sum_{j} f_{j}\left(A_{j}\right)=\frac{1}{2 \pi i} \int_{\partial \Pi\left(\frac{1+\varepsilon}{\varepsilon}\right)} \sum_{j} f_{j}(z) R\left(z, A_{j}\right) d z=\frac{1}{2 \pi i} \int_{\operatorname{Im} z=\left(\frac{1+\varepsilon}{\varepsilon}\right)} \sum_{j} f_{j}\left(z^{2}\right) 2 z R\left(z^{2},\left(B_{j}\right)^{2}\right) d z \\
=\frac{1}{2 \pi i} \int_{\operatorname{Im} z=\left(\frac{1+\varepsilon}{\varepsilon}\right)} \sum_{j} f_{j}\left(z^{2}\right)\left[R\left(z, B_{j}\right)-R\left(-z, B_{j}\right)\right] d z \\
=\frac{1}{2 \pi i} \int_{\left[\operatorname{Im} z=\left(\frac{1+\varepsilon}{\varepsilon}\right)\right]} \sum_{j} f_{j}\left(z^{2}\right)\left[R\left(z, B_{j}\right) d z=\sum_{j}\left[f_{j}\left(z^{2}\right)\right]\left(B_{j}\right)\right.
\end{gathered}
$$

with the appropriate orientations of the contours.

If we shift a parabola-type the sequence of operators far enough to the right, we obtain a sectorial sequence of operators (of arbitrary small angle) (see, e.g.,[9]). 


\section{Proposition 7.6}

Let $\varepsilon>-1$ and let $A_{j}$ be the sequence of operators on the Banach space X such that

$$
\sum_{j}\left\|R\left(\mu^{j}, A_{j}\right)\right\| \leq \sum_{j} \frac{M}{\left.\sqrt{\left|\mu^{j}\right|}\left(\left|\operatorname{Im} \sqrt{\mu^{j}}\right|\right)-(1+\varepsilon)\right)}\left(\mu^{j} \notin \overline{\Pi_{(1+\varepsilon)}}\right)
$$

Let $\left(B_{j}\right)_{\theta}:=A_{j}+((1+\varepsilon) / \cos \theta)^{2}$. Then $\left(B_{j}\right)_{\theta}$ is sectorial of angle $\pi / 2-\theta$. For $\theta=0$ onehas even

$$
\sum_{j}\left\|R\left(\mu^{j},\left(B_{j}\right)_{0}\right)\right\| \leq 2 M \sum_{j} \frac{1}{\left|\operatorname{Re} \mu^{j}\right|}\left(R r \mu^{j}<0\right)
$$

whence $-\left(B_{j}\right)_{0}$ is actually of half-plane type 0 .

\section{Proof}

First prove the assertion for $\left(B_{j}\right)_{0}$. Let $\operatorname{Re} \mu^{j}<0$ and find $(1+\varepsilon)=\mathrm{x}+$ iy such that

$(1+\varepsilon)^{2}=\mu^{j}-(1+\varepsilon)^{2}$ and $\mathrm{y}>(1+\varepsilon)$. By assumption on $\mu^{j}, \mathrm{x}^{2}<\mathrm{y}^{2}-(1+\varepsilon)^{2}$. Then

$$
\sum_{j}\left\|R\left(\mu^{j},\left(B_{j}\right)_{0}\right)\right\|=\sum_{j}\left\|R\left((1+\varepsilon)^{2}, A_{j}\right)\right\| \leq \frac{M}{\sqrt{x^{2}+y^{2}}(y-(1+\varepsilon))}
$$

But $\operatorname{Re} \mu^{j}=\operatorname{Re}(1+\varepsilon)^{2}+(1+\varepsilon)^{2}=-\left(\mathrm{y}^{2}-(1+\varepsilon)^{2}-\mathrm{x}^{2}\right)$, and hence

$\frac{\left|\operatorname{Re} \sum_{j} \mu_{j}\right|}{\sqrt{x^{2}+y^{2}}(y-(1+\varepsilon))}=\frac{y^{2}-(1+\varepsilon)^{2}-x^{2}}{\sqrt{x^{2}+y^{2}}(y-(1+\varepsilon))} \leq \frac{y^{2}-(1+\varepsilon)^{2}}{y(y-(1+\varepsilon))}=\frac{y+(1+\varepsilon)}{y}=1+\frac{y}{(1+\varepsilon)} \leq 2$

Fix $\theta \in(0, \pi / 2)$. Establish the estimate

$$
\sum_{j}\left\|R\left(\mu^{j}, e^{i \theta}\left(B_{j}\right)_{\theta}\right)\right\| \leq \frac{2 M}{(\operatorname{Cos} \theta)\left|\sum_{j} \operatorname{Re} \mu^{j}\right|}\left(\operatorname{Re} \mu^{j}<0\right)
$$

and this implies what stated in the proposition. Let $\operatorname{Re} \mu^{j}<0$ and find $(1+\varepsilon)=\mathrm{x}+\mathrm{iy}$ such that $\mathrm{y}>(1+\varepsilon)$ and $(1+\varepsilon)^{2}=\left(e^{-i \theta}\right) \mu^{j}-((1+\varepsilon) / \cos \theta)^{2}$.

Then $\left.\mu^{j}=e^{i \theta}\left((1+\varepsilon)^{2}+(1+\varepsilon) / \cos \theta\right)^{2}\right)$ and so

$$
\begin{aligned}
\frac{-\operatorname{Re} \mu^{j}}{\cos \theta}=y^{2}- & ((1+\varepsilon) / \cos \theta)^{2}-x^{2}+(\tan \theta) 2 x y \\
& =\left(y^{2}-(1+\varepsilon)^{2}\right)\left(1+\tan ^{2} \theta\right)-(x-y \tan \theta)^{2}
\end{aligned}
$$

and this i $\varepsilon>0$ by hypothesis. All in all we can estimate

$$
\begin{gathered}
\sum_{j}\left\|R\left(\mu^{j}, e^{i \theta}\left(B_{j}\right)_{\theta}\right)\right\|=\sum_{j}\left\|R\left((1+\varepsilon)^{2}, A_{j}\right)\right\| \leq \frac{M}{|(1+\varepsilon)|(\operatorname{Im}(1+\varepsilon)-(1+\varepsilon))} \\
=\sum_{j} \frac{M \cos \theta}{\left|\operatorname{Re} \mu^{j}\right|} \frac{\left(-\operatorname{Re} \mu^{j} / \cos \theta\right)}{\sqrt{x^{2}+y^{2}}(y-(1+\varepsilon))} \\
\frac{M \cos \theta}{\sum_{j}\left|\operatorname{Re} \mu^{j}\right|}\left[\frac{\left(y^{2}-(1+\varepsilon)^{2}\right)\left(1+\tan ^{2} \theta\right)-(x-y \tan \theta)^{2}}{\sqrt{x^{2}+y^{2}}(y-(1+\varepsilon))}\right] \\
\leq \frac{M \cos \theta}{\left|\operatorname{Re} \sum_{j} \mu^{j}\right|}\left[\frac{(y+(1+\varepsilon))\left(1+\tan ^{2} \theta\right)}{\sqrt{x^{2}+y^{2}}}\right] \leq \frac{2 M}{(\cos \theta)\left|\operatorname{Re} \sum_{j} \mu^{j}\right|}
\end{gathered}
$$

This concludes the proof.

One can easily show that a composition rule $f_{j}\left(\left(B_{j}\right)_{\theta}\right)=\left[f_{j}\left(\mathrm{z}+((1+\varepsilon) / \cos \theta)^{2}\right)\right]\left(A_{j}\right)$ holds when $f_{j}\left(\left(B_{j}\right)_{\theta}\right)$ is defined by the functional calculus for sectorial sequence of operators (oral so, in the case $\theta=$ 
0, by the one for half plane-type operators). By [7, Proposition1.3.6] one needs to know it only for functions $f_{j}$ belonging to a generating set for the primary calculus for $\left(B_{j}\right)_{\theta}$. Here the assertions follows from Cauchy's theorem.

How can access cosine sequence of operators functions by the functional calculus? Let $A_{j}$ be of parabolatype $\varepsilon>-1$. Consider the functions $\left(f_{j}\right)_{(1+\varepsilon)}(z)=\cos ((1+\varepsilon) \sqrt{z})((1+\varepsilon) \in \mathbb{R})$ which are bounded holomorphic functions on every parabola $\Pi_{\varphi_{j}}, \varphi_{j}>0$. So, $\operatorname{Cos}_{A_{j}}(1+\varepsilon):=\left(f_{j}\right)_{(1+\varepsilon)}\left(A_{j}\right)((1+\varepsilon) \in \mathbb{R})(2)$ is a well-defined family of closed sequence of operators, and it is no surprise that the analogue of Proposition 2.4 holds.

\section{Proposition 7.7}

Let $A_{j}$ be sequence of operators of parabola-type $\varepsilon>-1$ on the Banach space X. Then $-A_{j}$ sequence of generates a cosine function $(\operatorname{Cos}(1+\varepsilon))_{(1+\varepsilon) \in \mathbb{R}}$ if and only if $A_{j}$ is densely defined and $\cos _{A_{j}}(1+\varepsilon)$ (defined by (2)) is bounded sequence of operators for each $(1+\varepsilon) \in \mathbb{R}$, satisfying

$\sup _{(1+\varepsilon) \in[0,2]} \sum_{j}\left\|\operatorname{Cos}_{A_{j}}(1+\varepsilon)\right\|<\infty$. In this case $\operatorname{Cos}(1+\varepsilon)=\operatorname{Cos}_{A_{j}}(1+\varepsilon),(1+\varepsilon) \in \mathbb{R}$.

\section{Proof.}

Suppose that $-A_{j}$ be the sequence of generates a cosine function Cos, fix $\mu^{j}>(1+\varepsilon)^{2}$, and let $\mathrm{x} \in \mathcal{D}\left(A_{j}\right)$. Then $\mathrm{x} \in \mathcal{D}\left(\operatorname{Cos}_{A_{j}}(1+\varepsilon)\right)$ for every $\varepsilon>-1$. One has

$\operatorname{Cos}_{A_{j}}(1+\varepsilon) \mathrm{x}=\left[\left(f_{j}\right)_{(1+\varepsilon)} /\left(\mu_{j}+\mathrm{z}\right)\right]\left(A_{j}\right)\left(\mu_{j}+A_{j}\right) \mathrm{x}$ and $(1+\varepsilon) \mapsto \operatorname{Cos}_{A_{j}}(1+\varepsilon) \mathrm{x}$ is continuous, by Lebesgue's theorem. Taking Laplace transforms and applying Fubini's theorem yields (with $\mathrm{y}:=\left(\mu^{j}+A_{j}\right) \mathrm{x}$ )

$$
\begin{aligned}
& \int_{0}^{\infty} e^{-(1+\varepsilon)(1+\varepsilon)} \sum_{j} \operatorname{Cos}_{A_{j}}(1+\varepsilon) \mathrm{xd}(1+\varepsilon) \\
& =\frac{1}{2 \pi i} \int_{\partial \Pi_{\left(\frac{1+\varepsilon}{\varepsilon}\right)}}\left(\int_{0}^{\infty} e^{-(1+\varepsilon)(1+\varepsilon)} \cos ((1+\varepsilon) \sqrt{z}) d(1+\varepsilon)\right) \sum_{j} \frac{1}{\mu^{j}+z} R\left(z, A_{j}\right) d z y \\
& =\frac{1}{2 \pi i} \int_{\partial \Pi_{\left(\frac{1+\varepsilon}{\varepsilon}\right)} \sum_{j} \frac{(1+\varepsilon)}{\left((1+\varepsilon)^{2}+z\right)\left(\mu^{j}+z\right)} R\left(z, A_{j}\right) d z y} \\
& =(1+\varepsilon)\left((1+\varepsilon)^{2}+A_{j}\right)^{-1}\left(\mu^{j}+A_{j}\right)^{-1} y=(1+\varepsilon) R\left((1+\varepsilon)^{2},-A_{j}\right) x
\end{aligned}
$$

for $(1+\varepsilon)>(1+\varepsilon)$. Uniqueness of Laplace transforms yields $\operatorname{Cos}_{A_{j}}(1+\varepsilon) \mathrm{x}=\operatorname{Cos}(1+\varepsilon) \mathrm{x}$,

$\varepsilon>-1$. Since $\mathcal{D}\left(A_{j}\right)$ is dense and $\operatorname{Cos}_{A_{j}}(1+\varepsilon)$ are closed sequence of operators,

$\operatorname{Cos}_{A_{j}}((1+\varepsilon) 1+\varepsilon)=\operatorname{Cos}(1+\varepsilon)$ are bounded sequence operators for every $\varepsilon>-1$.

Conversely, suppose that $\operatorname{Cos}_{A_{j}}(1+\varepsilon)$ are bounded sequence of operators for every $\varepsilon>-1$ and that $\sup _{(1+\varepsilon) \in[0,2]} \sum_{j}\left\|\operatorname{Cos}_{A_{j}}(1+\varepsilon)\right\|<\infty$. By general functional calculus, $\operatorname{Cos}_{A_{j}}$ satisfies the cosine law [1, (3.88)], and so [1, Lemma 3.14.3] shows that $\operatorname{Cos}_{A_{j}}$ is exponentially bounded. As seen above, $\operatorname{Cos}_{A_{j}}$ $(1+\varepsilon) \mathrm{x}$ is continuous for all $\mathrm{x} \in \mathcal{D}\left(A_{j}\right)$, hence by the density of $\mathcal{D}\left(A_{j}\right)$, this is true even for all $\mathrm{x} \in \mathrm{X}$. Hence, $\operatorname{Cos}_{A_{j}}$ is cosine function. But the computation above (together with density of $\mathcal{D}\left(A_{j}\right)$ ) shows that the Laplace transform of $\operatorname{Cos}_{A_{j}}$ is $(1+\varepsilon) \mathrm{R}\left((1+\varepsilon)^{2},-A_{j}\right)$, whence $-A_{j}$ are the the sequence of generators of $\operatorname{Cos}_{A_{j}}$. 
Using a Convergence Lemma for the functional calculus on parbolas, one can aim for rational approximation results for cosine functions. Similarly, a Trotter-Kato type result holds.

\section{Acknowledgments}

We would like to thank our colleagues for interesting discussions and helpful ideas. Moreover, we thank the anonymous referee for suggestions to improve the paper.

\section{REFERENCES}

1. Wolf gang Arendt, Charles J. K. Batty, Matthias Hieber, Frank Neubrander. Vector Valued Laplace Transforms and Cauchy Problems. Monographs in Mathematics 96. Basel:Birkhauser. xi, 523 p,2001.

2. John B, Conway. A course in Functional Analysis $.2^{\text {nd }}$. Graduate Texts in Mathematics, 96. New York etc, Springer-Verlag. xvi, 399 p,1990.

3. Klaus-Jochen Engel, Rainer Nagel. One-Parameter Semigroups for linear Evolution Equations, Springer. Xxi ,586 p., 2000.

4. Markus Haase. Spectral properties of operator logarithms. Math.Z., 245(4): 761-779, 2003.

5. Markus Haase. Spectral mapping theorems for holomorphic functional calculi. J. London Math, Soc. (2), 71(3): 723-739, 2005.

6. Markus Haase. Functional calculus for groups and applications to evaluations.Submitted, 2006.

7. Markus Haase.The Functional Calculus for Sectorial Operators, Birkhauser-Verlag, Basel, 2006.

8. Sylvie Monniaux. A new approach to the Dore-Vennitheorem. Math. Nachr., 204:163-183,1999.

9. Markus Haase.Semigroup theory via functional calculus, 2006.

Citation: Simon Joseph, Isra Mukhtar, Manal Juma. "The Series of Semigroup Theory Via Functional Calculus", American Research Journal of Mathematics. vol 4, no. 1, 2018, pp. 1-17.

Copyright (C) 2018 Simon Joseph, Isra Mukhtar, Manal Juma. This is an open access article distributed under the Creative Commons Attribution License, which permits unrestricted use, distribution, and reproduction in any medium, provided the original work is properly cited. 